\title{
AIR2, un robot parallèle à actionnement pneumatique à deux degrés de liberté pour les applications de prise et dépose d'objets
}

\author{
Lotfi Chikh ${ }^{1, a}$, Cédric Baradat ${ }^{1}$, Philippe Poignet ${ }^{2}$, François Pierrot ${ }^{2}$ \\ ET MiCAËL MiCHELIN ${ }^{1}$ \\ 1 TECNALIA, Cap Omega, Rd-Pt B. Franklin, 34960 Montpellier, France \\ 2 LIRMM, Département de Robotique, 161 rue Ada, 34095 Montpellier, France
}

Reçu le 22 avril 2011, accepté le 11 mai 2011

\begin{abstract}
Résumé - Un prototype de robot parallèle actionné pneumatiquement à deux degrés de liberté a été développé afin de rendre accessible aux petites et moyennes entreprises la robotisation de tâches qui nécessiteraient le déplacement de charges de 5 à $20 \mathrm{~kg}$ en utilisant des vérins standards moins onéreux que les moteurs électriques. Après une présentation de la structure mécanique du robot ainsi que les modèles de ses actionneurs, la stratégie de commande innovante (commande prédictive généralisée avec boucle interne de force $\mathcal{H}_{\infty}$ ) est introduite, puis les résultats obtenus expérimentalement sont présentés et analysés.
\end{abstract}

Mots clés : Robot parallèle / pneumatique / commande

\begin{abstract}
A two degrees-of-freedom pneumatically driven parallel robot has been developed for pickand-place of payloads comprised between 5 and $20 \mathrm{~kg}$ using standard double acting cylinders cheaper than electrical motors. After the general presentation of the robot, the actuator models are developed and a novel control strategy is introduced based on a cascade scheme with a predictive position controller and an inner $\mathcal{H}_{\infty}$ force control. Some experimental results are then presented and analyzed in order to show the effectiveness of the controllers.
\end{abstract}

Key words: Parallel robotics / pneumatic actuators / modeling

\section{Introduction}

Les robots parallèles sont de plus en plus utilisés en industrie pour les tâches de prise et dépose d'objets du fait de leurs performances en termes de rapidité et précision. Cependant, leurs prix restent assez élevés du fait du coût important des moteurs électriques utilisés. De plus, les couples générés sont faibles et limitent la capacité des robots à transporter des charges élevées.

De leur côté, les actionneurs pneumatiques sont des actionneurs à bas coût ayant des rapports poids puissance importants ce qui les rend particulièrement avantageux dans les tâches de prise et dépose d'objets de poids intermédiaires à prix bas. C'est pour cette raison que l'objectif de cette étude est la conception et la commande d'un robot parallèle actionné pneumatiquement pour la manipulation d'objets de 5 à $20 \mathrm{~kg}$. À notre connaissance, il n'existe pas en industrie de robots parallèles à actionnement pneumatique. Ceci est dû principalement à

a Auteur pour correspondance : lotfi.chikh@tecnalia.com la difficulté de commander les actionneurs pneumatiques avec précision et robustesse [1,2] du fait des fortes nonlinéarités qui les caractérisent (compressibilité de l'air, valve et frottements importants).

Dans cet article, après la présentation et la description du prototype de robot réalisé, ses modèles sont développés puis une nouvelle stratégie de commande en cascade position-force combinant la commande prédictive et la commande robuste $\mathcal{H}_{\infty}$ sera présentée.

\section{Présentation du robot}

Le robot présenté dans cette étude est baptisé AIR2 (Robot à AIR à 2 degrés de liberté). Il est représenté dans la figure 1a. L'architecture du robot est identique à celle du robot PAR2 développée par Tecnalia et le Lirmm $[3,4]$ dans le but de générer deux translations dans le plan vertical. Son originalité consiste en l'utilisation de deux chaînes cinématiques passives dont le mouvement 


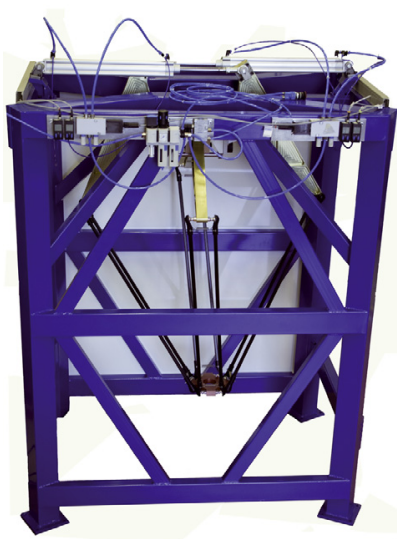

(a) Photo du robot AIR2

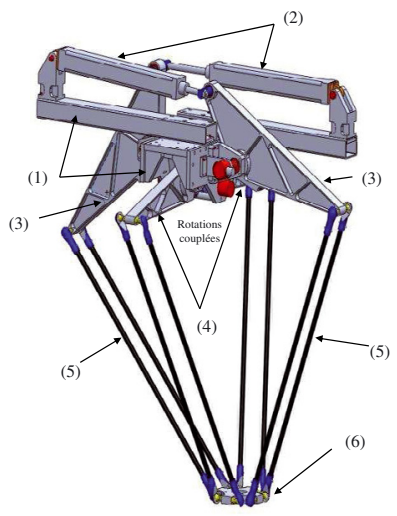

(b) Représentation $\mathrm{CAO}$

Fig. 1. Présentation du robot AIR2.

est couplé de telle sorte qu'elles garantissent un mouvement symétrique par rapport au plan du mouvement.

La représentation CAO de AIR2 (Fig. 1b) montre ses différents composants. Deux vérins standard ${ }^{1}$ à bas coût (2) remplacent les moteurs électriques qui sont habituellement utilisés. Ces vérins actionnent les bras actifs du robot (3). Leur placement est choisi de telle sorte que l'encombrement soit le plus réduit possible et a obéi à deux impératifs : (a) limiter leur rotation et donc l'influence qu'elle peut avoir sur la dynamique du robot, et (b) éviter les accélérations tangentielles trop importantes des vérins qui pourraient altérer les joints d'étanchéité. Deux autres bras passifs (4) sont couplés mécaniquement. Ils sont fixés à la base du robot (1) via des articulations passives. La nacelle du robot (6) est reliée aux bras actifs par un mécanisme à 4 barres (5) qui permet de contraindre la nacelle à bouger uniquement dans le plan horizontal.

\section{Modélisation}

Les paramètres géométriques du robot AIR2 sont représentés sur la figure 2 . Les modèles géométriques du

\footnotetext{
1 DNCB-50-400-PPV-A
}

robot AIR2 sont quasiment équivalents à ceux du robot PAR2 [3].

La partie pneumatique du robot comprend la valve et le vérin à double effet. La pression d'alimentation $p_{s}$ est supposée constante. La pression atmosphérique est notée $p_{0}$. Toute variation de volume de la chambre ou de la pression est décrite par la loi polytropique suivante [5] :

$$
p_{1} V_{1}^{\gamma}=p_{2} V_{2}^{\gamma}
$$

où $p_{i}$ est la pression dans l'une des deux chambres (les indices 1 et 2 sont relatifs à deux états de pression) et $V_{i}$ est le volume dans la chambre $i, \gamma$ est la constante polytropique. L'équation des gaz parfaits est donnée par :

$$
m=\frac{p V}{r T}
$$

où $m$ est la masse du gaz dans chaque chambre du vérin, $T$ est la température supposée constante (car l'air est constamment en circulation et est donc régénéré continuellement) et $r$ est la constante spécifique des gaz parfaits. En combinant les équations (1) et (2), l'expression de la dynamique des pressions est obtenue :

$$
\frac{\mathrm{d} p}{\mathrm{~d} t}=\frac{\gamma}{V(s)}\left[r T q_{m}(u, p)-p \frac{\mathrm{d} V}{\mathrm{~d} s} \dot{s}\right]
$$

où $u$ représente l'entrée de commande de la valve, $s$ est la position du piston dans le cas des cylindres. $q_{m}(u, p)$ représente le débit massique $\left(\frac{\mathrm{d} m}{\mathrm{~d} t}=q_{m}(u, p)\right)$ qui est fonction de la pression et de la tension d'entrée. La relation entre le volume et la position $s$ du piston du vérin est donnée par : $V_{i}(s)=V_{i}(0) \pm A_{i} s$ où $V_{i}(0)=A_{i} \frac{l}{2}$ est le volume initial de la chambre $i$ (la position initiale est le milieu du vérin). $l$ représente la course du vérin. $A_{i}$ est la section du piston des deux côtés de la chambre. Le modèle de la valve a été approximé - après identification au sens des moindres carrés - par l'expression suivante (plus de détails dans [6]) :

$$
q_{m}(u, p)=\varphi(p)+\psi(p) u
$$

$\varphi$ et $\psi$ sont des fonctions polynômiales d'ordre 5 en $p$. Ces modèles seront utilisés dans la commande du robot et notamment pour la linéarisation des équations de pression.

\section{Stratégie de commande}

En vue de commander le robot AIR2, la stratégie de commande décrite sur la figure 4 a été développée. Les trajectoires désirées $\left(x^{\text {ref }}, z^{\text {ref }}\right)$ sont spécifiées dans l'espace opérationnel. En utilisant le modèle géométrique inverse, les références angulaires $q_{1}^{\text {ref }}$ et $q_{2}^{\text {ref }}$ sont calculées et utilisées comme trajectoire de référence pour la commande dans l'espace articulaire implémentée pour chaque vérin. Les angles $q_{1}^{\text {mes }}$ et $q_{2}^{\text {mes }}$ sont mesurés à l'aide de codeurs incrémentaux et à partir de ces mesures, les poses sont calculées à l'aide du modèle géométrique directe du robot. 


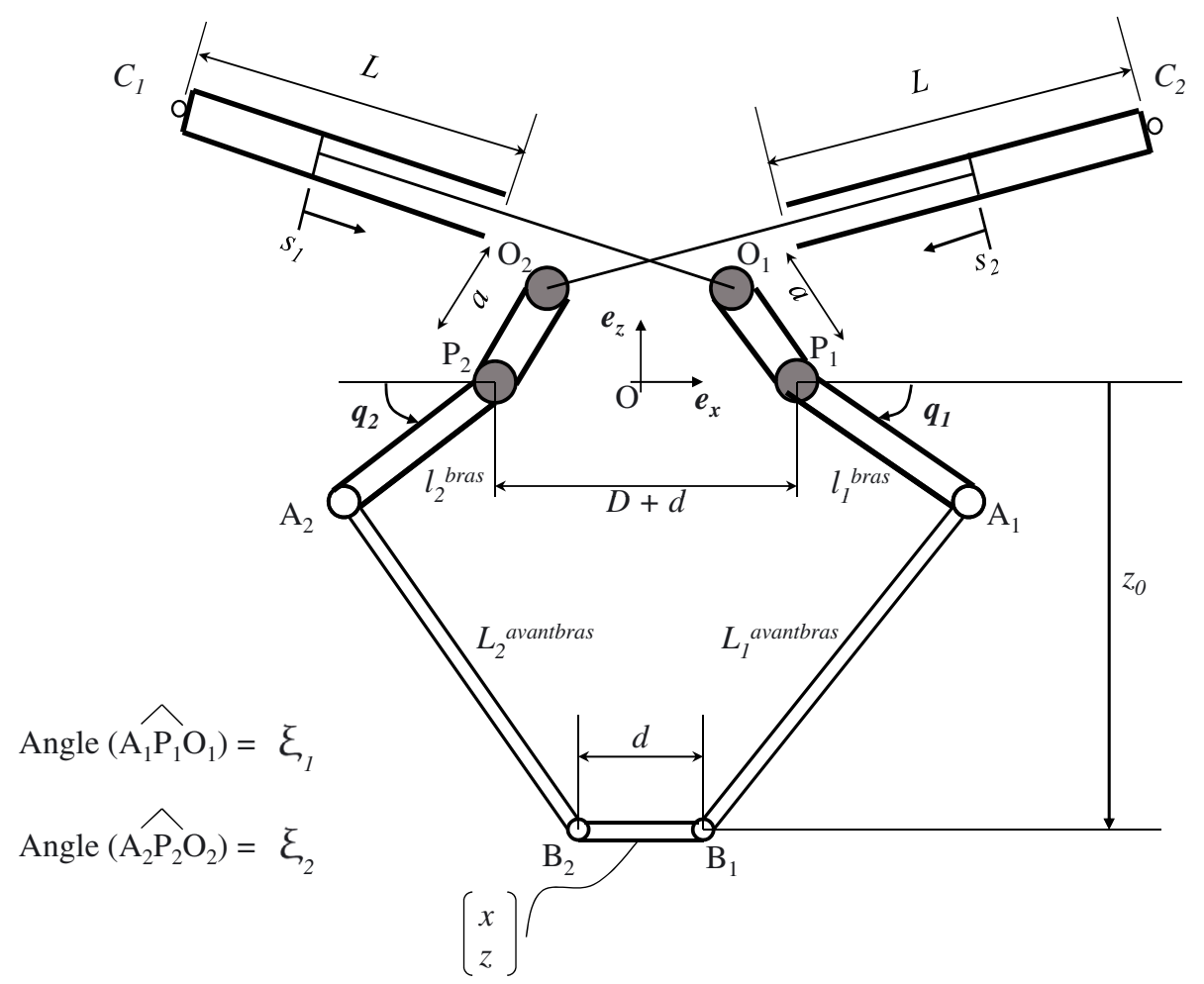

Fig. 2. Paramètres géométriques du robot AIR2.

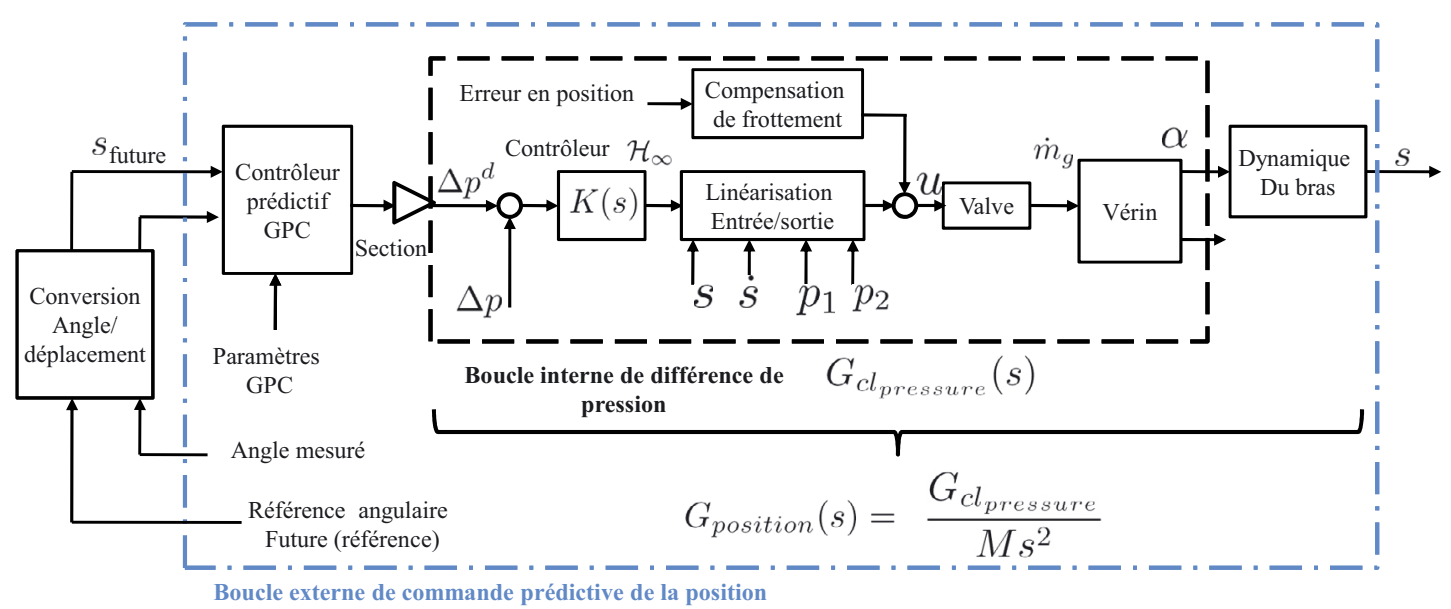

Fig. 3. Stratégie de commande en cascade GPC- $\mathcal{H}_{\infty}$ pour les vérins pneumatiques.

La stratégie de commande de la partie pneumatique du robot est résumée dans la figure 3. Elle est présentée en détail dans [7]. Elle consiste en un schéma en cascade position-force appliqué aux actionneurs après une linéarisation entrée/sortie. Pour le contrôleur de position, une commande prédictive généralisée (GPC) a été synthétisée. La commande prédictive a fait ses preuves en industrie mais a été peu utilisée en pneumatique. Elle a été implémentée dans des applications de robotique parallèle actionnée électriquement dans $[8,9]$ montrant d'excellentes performances en termes de suivi rapide et de robustesse par rapport aux variations de charge. D'autres applications en robotique peuvent être trouvées dans [10]. Pour la boucle interne, la force est contrôlée indirectement en mesurant et commandant la différence de pression, ellemême proportionnelle à la force. Un contrôleur $\mathcal{H}_{\infty}$ a été synthétisé après application d'une linéarisation exacte en utilisant les équations de pression du système. Le choix du contrôleur $\mathcal{H}_{\infty}$ permet d'assurer un bon suivi en dépit des incertitudes dues aux modèles et à la linéarisation exacte ainsi qu'aux variations de charge qui peuvent être importantes.

\section{Expérimentations}

Afin de valider la stratégie de commande proposée, trois tests sont effectués sur le robot : un cycle de prise et 


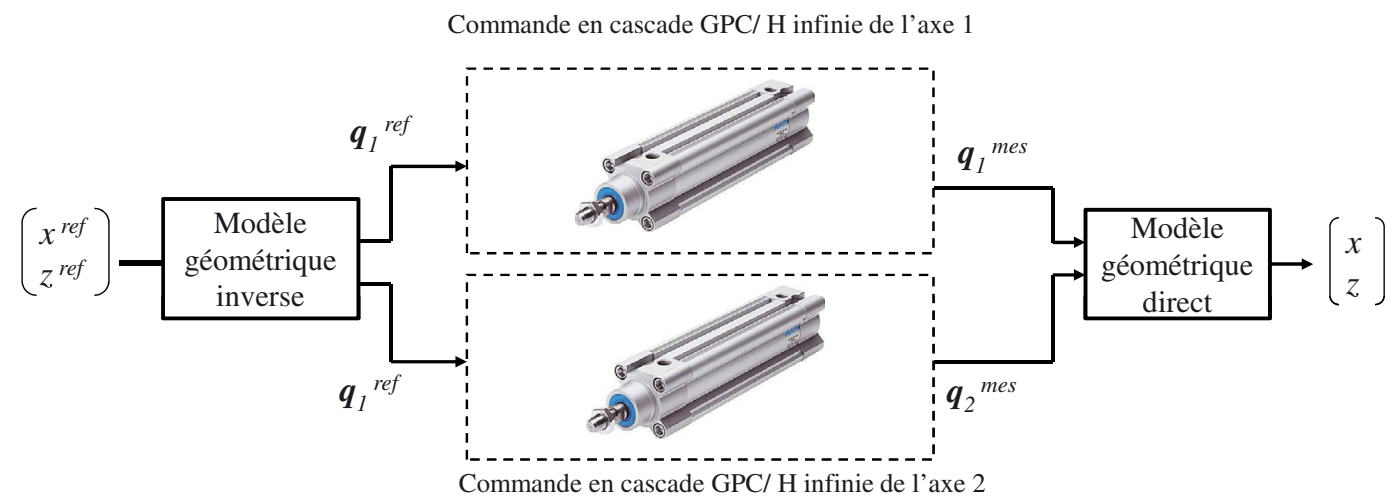

Fig. 4. Schéma de commande du robot AIR2.
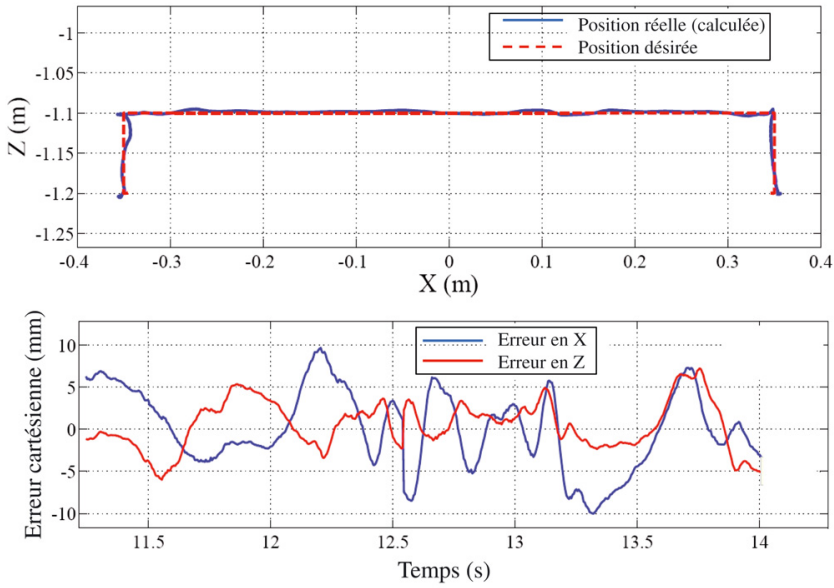

Fig. 5. Trajectoire de prise et dépose d'objet effectuée à vide d'une durée de $2,9 \mathrm{~s}$.

dépose d'objets à vide (sans charge), un cycle de prise et dépose d'objet avec une charge de $15 \mathrm{~kg}$ et enfin une trajectoire de référence circulaire avec une charge de $20 \mathrm{~kg}$.

Le premier test est un cycle de prise et dépose d'objet de $100 \times 700 \times 100 \mathrm{~mm}$ avec une accélération de $2 \mathrm{~g}$. Le résultat présenté à la figure 5 montre que l'erreur de suivi maximale est égale à $10 \mathrm{~mm}$ et le cycle est effectué en moins de $3 \mathrm{~s}$. En augmentant la charge (test à $15 \mathrm{~kg}$ représenté sur la Fig. 6), l'accélération testée est diminuée pour éviter une saturation des signaux de commande. Le suivi est toujours de bonne qualité même si une légère augmentation de la valeur de l'erreur maximale est observée $(12 \mathrm{~mm})$. Enfin le dernier test montre le suivi d'une référence circulaire pour une valeur de la charge égale à $20 \mathrm{~kg}$. L'erreur maximale de suivi est inférieure à $8 \mathrm{~mm}$.

\section{Conclusion}

AIR2, un prototype de robot parallèle actionné pneumatiquement à deux degrés de liberté a été présenté et modélisé. Deux chaînes cinématiques fermées passives sont rajoutées aux chaînes actives dans un plan normal
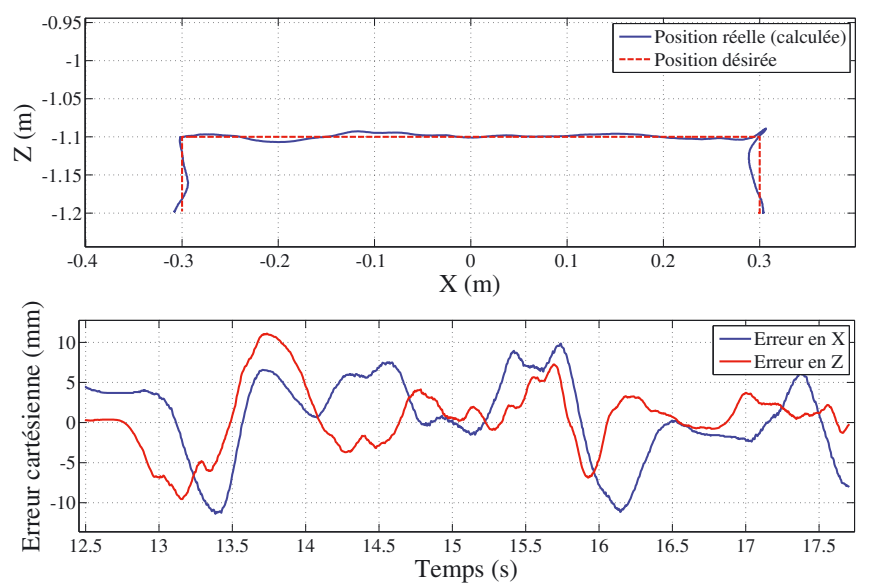

Fig. 6. Mouvement de prise et dépose d'une charge de $15 \mathrm{~kg}$.
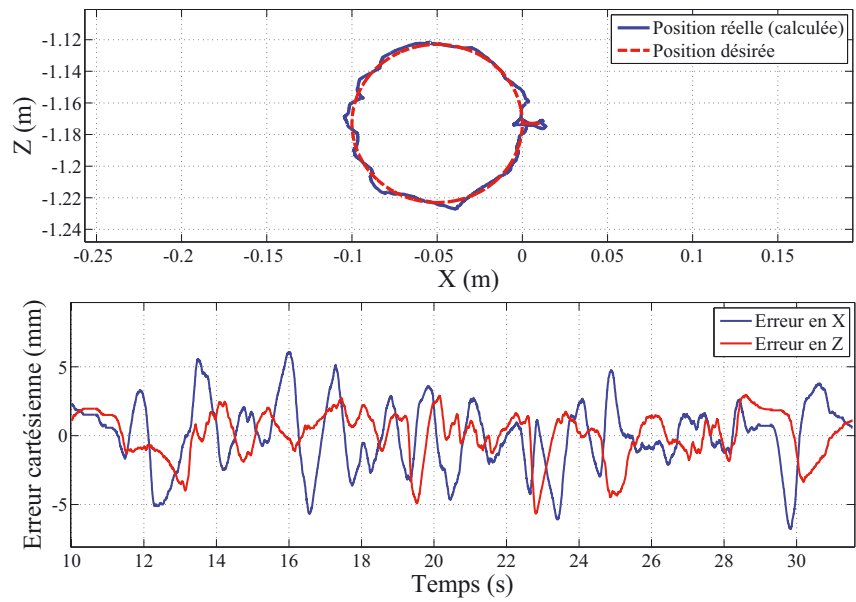

Fig. 7. Trajectoire de référence circulaire avec une charge de $20 \mathrm{~kg}$.

au plan de travail et fournissent les dernières contraintes à la plate-forme pour ne laisser que les deux degrés de liberté du mouvement. Une stratégie de commande en cascade position-force qui combine la commande prédictive et la commande robuste $\mathcal{H}_{\infty}$ a été implémentée expérimentalement. Les résultats obtenus montrent que 
l'utilisation des actionneurs pneumatiques pour les robots parallèles peut être une alternative sérieuse aux moteurs électriques coûteux et aux faibles rapports poids puissance. Les tests expérimentaux ont montré des résultats de commande encourageants en terme de suivi rapide de trajectoire et de capacité du robot à déplacer des charges allant jusqu'à $20 \mathrm{~kg}$.

\section{Références}

[1] X. Brun, M. Belgharbi, S. Sesmat, D. Thomasset, S. Scavarda, Control of an electropneumatic actuator, comparison between some linear and nonlinear control laws, J. Syst. Control Eng., Special Issue Controls in Fluid Power Systems 123 (1999) 387-404

[2] A. Girin, F. Plestan, X. Brun, A. Glumineau, High-order sliding mode controllers of an electropneumatic actuator: Application to an aeronautic benchmark, IEEE Trans. Control Sys. Technol. 17 (2009) 633-645

[3] C. Baradat, V. Nabat, O. Company, S. Krut, F. Pierrot, Par 2: a spatial mechanism for fast planar, 2-dof, pick-and-place applications, Proc. Second International Workshop on Fundamental Issues and Future Research Directions for Parallel Mechanisms and Manipulators, Montpellier, 2008
[4] F. Pierrot, C. Baradat, V. Nabat, S. Krut, M. Gouttefarde, Above 40g acceleration for pick-and-place with a new 2-dof pkm. In IEEE International Conference on Robotics and Automation, Kobe, Japan, 2009

[5] A. Hildebrandt, O. Sawodny, R. Neumann, A cascaded tracking control concept for pneumatic muscle actuators, in European Control Conference 2003 (ECC03), Cambridge UK, (CD-Rom), 2003

[6] M. Belgharbi, S. Sesmat, S. Scavarda, D. Thomasset, Analytical model of the flow stage of a pneumatic servodistributor for simulation and nonlinear control, in The Sixth Scandinavian International Conference on Fluid Power, Tampere-Finlande, 1999, pp. 847-860

[7] L. Chikh, P. Poignet, F. Pierrot, C. Baradat, A mixed GPC- $\mathcal{H}_{\infty}$ robust cascade position-pressure control strategy for electropneumatic cylinders, in IEEE International Conference on Robotics and Automation, Anchorage, Alaska, 2010

[8] A. Vivas, P. Poignet, Model based predictive control of a fully parallel robot, Symposium on Robot Control, Wroclaw (Poland), 2003

[9] A. Vivas, P. Poignet, Predictive functional control of a parallel robot, Control Eng. Pract. 13 (2005) 863-874

[10] J. Richalet, R. Abu, H.B. Kuntze, A. Jacubasch, W. Schill, Predictive functional control, application to fast and accurate robot, Tenth IFAC world congress, Munich, Germany, 1997 